\title{
OUTBURST ACTIVITY DATA ON SELECTED CATACLYSMIC VARIABLES
}

\author{
T. VANMUNSTER ${ }^{1}$, S. B. HOWELL ${ }^{2}$ \\ 1. Vereniging voor Sterrenkunde, Werkgroep Veranderlijke \\ Sterren, Walhostraat 1A, B-3401 Landen, Belgium \\ 2. Planetary Science Institute, Astrophysics Group, 620 N. 6th \\ Ave, Tucson, AZ 85705, USA
}

\begin{abstract}
We have created a database with outburst activity for 269 selected cataclysmic variables. It includes nearly 1000 outburst measurements discovered through 1995 June. The database and a complete paper describing the observations and the sources of information that were consulted are accessible over the Internet.
\end{abstract}

\section{Introduction and history}

The Outburst Activity Database on Selected Cataclysmic Variables originated in 1992 as an attempt to collect extensive details of recent outburst data for dwarf novae, observed by members of the Belgian Astronomical Association VVS (Vereniging voor Sterrenkunde) working group 'Veranderlijke Sterren'. The objective was to focus this database and to establish a list of dwarf novae for which little or no observational data existed. After a few months of operation, we learned about similar initiatives, set up by amateur astronomers in other countries, the most notable being the 'Recurrent Objects Catalogue' issued by Hurst (1989). Two limitations of this database are: (i) the database contains only a few objects with a negative declination and (ii) the object must be visible (at maximum magnitude) in large amateur telescopes (i.e. become brighter than visual magnitude 15 or so). However, some exceptions to these rules do exist in the database.

\section{Structure of the database}

The Outburst Activity Database currently contains nearly 1000 entries on 269 selected cataclysmic variables. For some objects, detailed outburst mea-

63

A. Evans and J. H. Wood (eds.), Cataclysmic Variables and Related Objects, 63-64.

(1) 1996 Kluwer Academic Publishers. Printed in the Netherlands. 
surements are available, whereas for others very little or no information at all could be retrieved. The database includes a large number of known and suspected tremendous outburst amplitude dwarf novae (TOADs; Howell, Szkody \& Cannizzo 1995). The tabular format, used to represent the outburst data, is described in detail in the internet version of this paper.

\section{Variable star alert programmes}

We conclude this short paper by briefly discussing the variable star alert programmes. The major contribution to visual observations of dwarf novae comes from a few very active and enthusiastic observers. Most of them are very experienced variable star observers, with proven skills in 'traditional' variable star work. Lots of practice and perseverance has turned them into well-trained amateurs, who are capable of memorising tens of star fields. On every clear night, they visually check these fields, hunting for 'new' stars. Using this technique, some amateurs are capable of visually inspecting between 50 and 100 star fields in one single night. Special observing programmes have been set up, to direct the attention of these amateurs towards the more exotic cataclysmic variables. Two such examples are discussed in the electronic version of this paper: the Recurrent Objects Programme (The Astronomer Group, UK) and the Cataclysmic Variables Alert Programme (VVS, Belgium). When activity of a suspected star is noticed, other members of the network are informed (by telephone) in order to obtain immediate confirmation. Positive identifications of outbursts result in alert calls (electronic circulars), that are issued to amateurs all over the world and to interested professional astronomers. The Outburst Activity Database and a detailed paper describing it are both available on line at the WWW site of the second author (homepage http://www.psi.edu/sbhgen.html).

Acknowledgements. We would like to thank Paul Van Cauteren, Director of the Belgian working group 'Veranderlijke Sterren' and Gary Poyner, Coordinator of the Recurrent Objects Programme, for their continuous support and cooperation. Special thanks also to the staff members of the Uccle Observatory, Belgium.

\section{References}

Howell, S., Szkody, P., Cannizzo, J., 1995, Ap. J., 439, 337

Hurst, G., 1989, UK Nova/Supernova Patrol, Recurrent Objects Catalogue, 1-7 